\title{
Concluding summaries
}




\title{
Observations Relating to Stellar Activity, Magnetism and Dynamos
}

\author{
C. Jordan \\ Department of Theoretical Physics, University of Oxford, 1 Keble Road, \\ Oxford OX1 3NP, UK
}

\begin{abstract}
Dynamo theory can in principle be tested by comparing its predictions with a variety of types of solar and stellar observations. The progress apparent from papers presented at this Colloquium is summarized, and areas where further observations would be of particular value are stressed.
\end{abstract}

\section{Introduction}

The aim of IAU Colloquium 130 was to bring together the scientists working on stellar dynamo theory with those making observations related to stellar activity. The Colloquium was timely in that there have been recent theoretical developments, particularly in the study of non-linear effects, and a wide range of observations is now possible, from X-ray to radio wavelengths. In particular, helioseismology is providing new ways of probing convection zone properties, and the techniques for determining stellar surface magnetic fields have substantially improved over the past few years. Here we examine the extent to which the existing observations can be used to confront dynamo theory, and what further observations are required.

\section{Internal conditions: the convection zone}

Theoretical arguments concerning the generation of magnetic fields, and the timescale for their emergence through the stellar surface, require the region of generation to be placed within about one pressure scale height above the base of the convection zone (Parker, 1975). The properties of the surface fields then depend on the rotation rate, and the rate of differential rotation, with depth in the convection zone. Until recently there was no way of making observations of 
these regions, but the situation has been changed significantly by the advent of helioseismology.

The papers by Goode and Paternó, for example, addressed this area. The global oscillations can be used to sample the region at $R>0.5 R_{\odot}$ and the splittings according to $l$ allow the differential rotation to be investigated. The high $l$ splittings reflect conditions near the surface, whilst the low $l$ splittings reflect conditions near the base of the convection zone. The available data sets (see Goode for references) show that near the equator the rotation rate decreases only slowly from the surface value until near the base of the convection zone. The data for non-equatorial latitudes are less reliable, but show tendencies that depart from the surface law. Belvedere et al. also use helioseismology data to deduce that the gradient of the angular velocity in the boundary layer at the base of the convection zone should change from positive to negative at a latitude of around $37^{\circ}$, and relate this result to observations of the latitude dependence of solar activity and to their non-linear dynamo model.

Near the base of the convection zone there seems to be a rapid transition to solid body rotation, but different data sets give conflicting evidence on the core rotation rate. However, the data support the concept of dynamo action near the base of the convection zone. On the other hand, they already exclude rotation on cylinders, predicted by some theories.

Future observations, both from space (e.g. from SOHO) and from groundbased networks, (e.g. GONG and the Birmingham network), should allow a wider variety of modes to be observed, and the accuracy of the data to be improved. Thus there is a real expectation of a secure knowledge of the solar differential rotation as a function of depth and latitude, and as a function of the solar activity cycle, in the not too distant future. These data will provide a foundation for extending dynamo theory to other stars.

\section{Solar surface magnetic fields}

Observations of solar magnetic fields at high spatial resolution also provide valuable boundary conditions to constrain dynamo theories, and several speakers addressed the interpretation of these data. For example, Ruzmaikin et al. analyzed magnetograms obtained at Kitt Peak and La Palma in terms of fractal structure within a turbulent cell, such as a supergranule. They show that such a fractal distribution can be produced in an inhomogeneous "stretch-twist-fold" dynamo model, and investigate the resulting degree of inhomogeneity in the velocity field.

Stenflo discussed synoptic observations obtained from Kitt Peak and Mount Wilson, and how they can be used to infer the rotational phase velocity. He finds that daily longitude displacements and the recurrence of patterns at the central meridian lead to completely different rotation laws. He suggests that these can be reconciled if the observed pattern of surface fields is renewed from deep layers in a time scale of less than a month, and again argues that this is evidence for dynamo 
action at the base of the convection zone. The quasi-rigid rotation found from the recurrence method would then represent the phase velocity of the source pattern.

Another way of studying the magnetic field and the velocity fields is to use measurements of the positions of sunspots as a function of time, a method pioneered by Tuominen (1945). Ribes et al. reported the results of analyzing spectroheliograms obtained at Meudon during solar cycle 21, using techniques involving digitized data (Mein and Ribes, 1990). They find that there is a zonal and timedependent meridional circulation, which coincides with the large scale azimuthal field as measured at Stanford by Hoeksema, and that the rotation rates for each year vary from the mean differential rotation. They interpret these results in terms of magnetic convective tori and azimuthal rolls, and suggest that behaviour of the latter could explain the torsional pattern observed by Howard and LaBonte (1980) and Snodgrass (1986) from measurements of velocity fields.

Although some of the observations discussed in Sections 2 and 3 lead to results that are preliminary, or tentative, they are clearly well worth pursuing. The studies of the magnetic fields, together with the helioseismological data, are revealing the complexity of the solar dynamo, and cast doubts on the simple $\alpha \Omega$ model. Parker (1987) has reviewed the difficulties produced by recent observations, in particular the sign of the migration of the azimuthal field in models where the the angular velocity decreases inwards, and discusses ways in which the simple model may have to be modified.

\section{Other solar observations}

It is now generally accepted that the energy emitted from the transition region and corona originates from the dissipation of magnetic energy. Although there may be a non-magnetic component to the energy deposited in the chromosphere, the correlation between the spatial location of strong regions of emission in chromospheric lines, such as $\mathrm{Ca}$ II $\mathrm{H}$ and $\mathrm{K}$, and regions of high magnetic flux, is taken to indicate heating via the magnetic field in this region too. Schrijver (1987) has shown that the "excess" flux (i.e. the component heated via the magnetic field) in the Ca II and $\mathrm{Mg}$ II emission lines correlates linearly with the flux in transition region lines, such as C IV, which are also stronger over the supergranulation boundaries. The overlying coronal emission does not show clearly the supergranulation pattern but is more amorphous (Reeves, 1976). A study of emission line intensities by Schrijver et al. (1985) showed tight correlations between transition region and coronal fluxes, in both the quiet sun and integrated active region fluxes. The coronal emission is related to the transition region emission approximately according to

$$
F(\text { Corona }) \propto F(\text { Transition Region })^{1.5}
$$

The same correlation is found for other cool stars which have X-ray emission. (See Section 5). These flux-flux correlations appear to be the result of the energy balance within the atmosphere (Jordan et al., 1987; Jordan, 1990). 
Shcherbakov and Shcherbakova discussed their observations of the He I 10830 $\AA$ absorption line, which is formed in the high chromosphere. These, and previous observations (see their paper for references), show that there is a good correlation between the depth of the He I line and the Ca II K index, and also with the Fe XIV coronal index. They give details on the variation of the He I line with the solar cycle. One interesting result (Sitnik et al., 1986) is that during the solar maximum the helium chromospheric network is enhanced, as well as there being additional contributions from active regions. Others, (e.g. Skumanich et al., 1984) have come to a similar conclusion from the variations of the $\mathrm{Ca} \mathrm{II} \mathrm{K}$ index.

The variation of chromospheric and coronal lines with the solar cycle, and as a function of the type of spatial structure observed, contains potentially a great deal of useful information on the magnetic fields that extend beyond the photosphere. Reviews of these data can be found in the Proceedings of COSPAR Symposium 12 (Chapman, 1988). The interpretation is at present limited by our lack of detailed knowledge concerning the processes heating the chromosphere and corona and their precise dependence on the magnetic field.

The emission line fluxes can also be correlated with the underlying photospheric magnetic field, and can be used to find the energy required to be balanced by the as yet unknown energy dissipation processes. Since the chromospheric and coronal magnetic fields must be related to the dynamo generated fields, correlations between the energy requirements and the solar cycle should eventually help to understand the solar dynamo.

\section{Stellar Observations}

Observations of the chromospheric and coronal properties of main sequence stars which have outer convection zones can provide important additional information on dynamo action. In particular, the dependence of emission line fluxes on $\Omega$, the stellar rotation rate, can be found for stars of similar spectral type and structure, as well as the dependence on spectral type, and hence convection zone structure, for stars of a similar $\Omega$. Rutten (1987) has carried out studies of this type using the $\mathrm{Ca}$ II flux and the $\mathrm{Ca}$ II "excess" flux. The Ca II flux shows a systematic dependence on $B-V$ for a given range of $\Omega$, but also shows the presence of a "minimum" flux at each $B-V$. When the Ca II excess flux is examined, it is found that, for a chosen range of $B-V$, the excess flux depends mainly on $\Omega$. Noyes et al. (1984) have also found that their Ca II index depends on the inverse Rossby number, although for main sequence stars of type G0 to K9 the dependence on just $\Omega$ is very similar. Using a simple power law fit the dependence is roughly

$$
\Delta F_{\mathrm{CaII}} \propto \Omega^{1.2}
$$

The X-ray fluxes show a stronger dependence on rotation, more like

$$
F_{X} \propto \Omega^{2}
$$


These different dependences on $\Omega$ suggest the presence of two separate energy dissipation processes for the chromosphere and corona. The dependence of the chromospheric and coronal magnetic fields on $\Omega$ can be found only if specific heating processes are postulated. (See, for example, Montesinos and Jordan, 1988; Jordan, 1990). Further chromospheric modelling and improved data for X-ray fluxes are required to pursue this approach in a more quantitative manner.

It is also vital to continue and improve the present work on measuring stellar magnetic fields and their dependence on the rotation rate. Saar reviewed recent progress. The sample available at present contains a mixture of observing methods and different degrees of sophistication in the modelling of the lines used. There are also uncertainties in separating the observed mean fields, $\langle B f\rangle$, into the values of $B$ and the filling factor $f$. At present the dependence of $\langle B f\rangle$ on $\Omega$ found by different authors (Schrijver et al., 1989; Saar, 1990) is between

$$
\langle B f\rangle \propto \Omega^{2} \text { and } \Omega^{1.3}
$$

A comparison between the observed dependence of $\langle B f\rangle$ on $\Omega$ and that predicted by a simple dynamo theory, was presented by Montesinos et al. In particular, the results of adopting two different variations of the angular velocity with depth, and two different ways of estimating the filling factor, $f$, were explored. The different approaches affect both the absolute values of $\langle B f\rangle$ at a given $\Omega$, and the dependence of $\langle B f\rangle$ on $\Omega$.

There is also considerable interest in Ap, CP and F stars, to investigate where convection zone dynamos take over from fossil fields (e.g. Landstreet; Khokhlova). Observations of magnetic fields on pre-main-sequence stars are also important in this respect (e.g. Basri and Marcy).

Studies of the rotation and activity of giant stars have begun, but the sample of single stars for which the essential data are known is rather small. The situation should improve when further observations become available fron ROSAT and the Hubble Space Telescope. The theory of dynamos in giant stars is also less advanced. However, Dravins et al. have studied $\beta$ Hydri (G2 IV) as an example of the postmain-sequence evolution of a solar type star and Gray presented evidence for a dynamo generated magnetic brake operating to cause a rapid loss of angular momentum between stars of type G0 III and G3 III. If stars cooler than G3 III have a unique dependence of rotation on spectral type then the inclination can be found and it can be deduced that the activity occurs towards equatorial latitudes. Changes in stellar structure, giving lower surface gravities, may then be the main cause of the absence of hot coronae for stars cooler than about K1 III.

The most direct information on stellar magnetic fields and star spots comes from observations of RS CVn and other binary systems. A number of papers were presented on methods of surface imaging (e.g. Piskunov; Hackman et al.) on results for particular systems. For example, Vogt and Hatzes find that in the system UX Arietis the spotted primary has an equatorial rotation rate which is synchronized to the orbital angular velocity, while the differential rotation is in the opposite sense to, and an order of magnitude smaller than, that of the Sun. In spite of the lower differential rotation the primary is very active. Zeilik finds that in several 
systems the active regions are clustered at two longitudes and tend to occur at high latitudes. This distribution may reflect the magnetic field interactions occurring in close binaries with synchronous rotation. The complexity of the interpretation is apparent from the work of Donati and Semel, who find that in $\mathrm{HR} 1099$ and $\sigma^{2} \mathrm{CrB}$ the magnetic field is correlated with bright photospheric regions rather than with dark spots. Schrijver and Zwaan (and Demircan and Derman) discussed the role of binarity in causing "overactivity" and suggest that this somehow enhances the dynamo action in the primary star. Hall has studied a large sample of other systems and finds that in many the differential rotation decreases rapidly with the Rossby number, and is not inversely proportional to it, as predicted by linear theory. This could be consistent with nonlinear theories where rapid rotation suppresses the differential rotation. These binary systems may eventually allow dynamo theory to be tested in situations very different from those in single main-sequence stars.

Finally, the interpretation of stellar UV and X-ray observations depends critically on the availability of accurate measurements of basic stellar parameters and distances; results from the Hipparcos satellite should eventually improve these data.

\section{References}

Chapman, G.A.: 1988, Editor, Adv. in Space Res. Vol. 8, No. 7.

Howard, R.H., LaBonte, B.J.: 1980, Astrophys. J. Letts. 239, L33.

Howard, R.H., Gilman, P.A., Gilman, P.I.: 1984, Astrophys. J. $283,373$.

Jordan, C.: 1990, In Proc. of Heidelberg Conf. on Mechanisms of Coronal Heating, June, 1990. In Press.

Jordan, C., Ayres. T.R., Brown, A., Linsky, J.L., Simon, T.: 1987, Mon. Not. R. astr. Soc. $225,903$.

Mein, P., Ribes, E.: 1990, Astron. Astrophys. 227, 577.

Montesinos, B., Jordan, C.: 1988, In A Decade of UV Astronomy with IUE, ESA SP-281, Vol. 1, 283.

Noyes, R.W., Hartmann, L.W., Baliunas, S.L., Duncan, D.K., Vaughan, A.H.: 1984, Astrophys. J. $279,763$.

Parker, E.N.: 1975, Astrophys. J. 198, 205.

Parker, E.N.: 1987, Sol. Phys. 110, 11.

Reeves, E.M.: 1976, Sol. Phys. 46, 53.

Rutten, R.G.M.: 1987, Astron. Astrophys. 177, 131.

Saar, S.H.: 1990, In Solar Photosphere: Structure, Convection and Magnetic Fields, Proc. IAU Symp. 138, ed. J.O. Stenflo, Kluwer, Dordrecht, p. 427.

Schrijver, C.J.: 1987, Astron. Astrophys. 172, 111.

Schrijver, C.J., Cote, J., Zwaan, C., Saar, S.H.: 1989, Astrophys. J. 337, 964.

Schrijver, C.J., Zwaan, C., Maxson, C.W., Noyes, R.W.: 1985, Astr. Astrophys. 149, 123.

Sitnik, G.F., Kozlova, L.M., Divlikeev, M.I., Porfiryeva, G.A.: 1986, Soviet Solar Data, No. 12,67 .

Skumanich, A., Lean, J.L., White, O.R., Livingston, W.C.: 1984, Astrophys. J. $282,776$.

Snodgrass, H.B.: 1986, Astrophys. J. Letts. 316, L91.

Tuominen, J.: 1945, The Observatory 66, 160. 